ELECTRONIC RESEARCH ANNOUNCEMENTS

OF THE AMERICAN MATHEMATICAL SOCIETY

Volume 10, Pages 155-158 (December 24, 2004)

S $1079-6762(04) 00140-4$

\title{
COUNTEREXAMPLES TO THE NEGGERS-STANLEY CONJECTURE
}

\author{
PETTER BRÄNDÉN
}

(Communicated by Sergey Fomin)

\begin{abstract}
The Neggers-Stanley conjecture asserts that the polynomial counting the linear extensions of a labeled finite partially ordered set by the number of descents has real zeros only. We provide counterexamples to this conjecture.
\end{abstract}

A finite partially ordered set (poset) $P$ of cardinality $p$ is said to be labeled if its elements are identified with the integers $1,2, \ldots, p$. We will use the symbol $\prec$ to denote the partial order on $P$ and $<$ to denote the usual order on the integers. The Jordan-Hölder set $\mathcal{L}(P)$ is the set of permutations $\pi=\left(\pi_{1}, \ldots, \pi_{p}\right)$ of $[p] \stackrel{\text { def }}{=}$ $\{1,2, \ldots, p\}$ which encode the linear extensions of $P$. More precisely, $\pi \in \mathcal{L}(P)$ if $\pi_{i} \prec \pi_{j}$ implies $i<j$.

A descent in a permutation $\pi$ is an index $i$ such that $\pi_{i}>\pi_{i+1}$. Let $\operatorname{des}(\pi)$ denote the number of descents in $\pi$. The $W$-polynomial of a labeled poset $P$ is defined by

$$
W(P, t)=\sum_{\pi \in \mathcal{L}(P)} t^{\mathrm{des}(\pi)} .
$$

$W$-polynomials appear naturally in many combinatorial contexts [2, 7, 8], and are connected to Hilbert series of the Stanley-Reisner rings of simplicial complexes [10. Section III.7] and algebras with straightening laws [9, Theorem 5.2.].

Example 1. Let $P_{2,2}$ be the labeled poset shown in Figure 1 Then

$$
\mathcal{L}\left(P_{2,2}\right)=\{(1,3,2,4),(1,3,4,2),(3,1,2,4),(3,1,4,2),(3,4,1,2)\},
$$

so $W\left(P_{2,2}, t\right)=4 t+t^{2}$.

$$
P_{2,2}=\left.\left.\right|_{1} ^{2} \backslash\right|_{3} ^{4}
$$

Figure 1. The poset $P_{2,2}$.

Received by the editors August 31, 2004.

2000 Mathematics Subject Classification. Primary 06A07, 26C10.

Key words and phrases. Neggers-Stanley conjecture, partially ordered set, linear extension, real roots.

(C)2004 American Mathematical Society Reverts to public domain 28 years from publication 
When $P$ is a $p$-element antichain, then $\mathcal{L}(P)$ consists of all permutations of $[p]$, and $W(P, t)$ is the $p$ th Eulerian polynomial. The Eulerian polynomials are known [3] to have only real zeros. In this instance, the Neggers-Stanley conjecture holds:

Conjecture 1 (Neggers-Stanley). For any finite labeled poset $P$, all zeros of the polynomial $W(P, t)$ are real.

A poset $P$ is naturally labeled if $i \prec j$ implies $i<j$. Conjecture 1 was made by J. Neggers [4] in 1978 for naturally labeled posets, and extended by R. P. Stanley in 1986 to arbitrary labelings. It has been proved in some special cases (see 2. 11]). A weaker unimodality property of $W$-polynomials was recently proved [6] (see also [1]) for graded naturally labeled posets.

In this note, we construct counterexamples to Conjecture 1utilizing the following construction. Let $\mathbf{m} \sqcup \mathbf{n}$ denote the disjoint union of the chains $1 \prec 2 \prec \cdots \prec m$ and $m+1 \prec m+2 \prec \cdots \prec m+n$. Let $P_{m, n}$ be the labeled poset obtained by adding the relation $m+1 \prec m$ to the relations in $\mathbf{m} \sqcup \mathbf{n}$; see Figure 2,

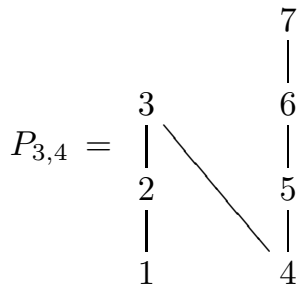

Figure 2. The poset $P_{3,4}$.

Theorem 1. Let $M$ be a positive integer. The polynomial $W\left(P_{m, n}, t\right)$ has more than $M$ non-real zeros provided $\min (m, n)$ is sufficiently large.

The posets $P_{m, n}$ are not naturally labeled, so the original conjecture of Neggers remains open.

The rest of the paper is devoted to the proof of Theorem 1 At the end, we discuss specific (minimal) counterexamples obtained from Theorem 1 .

Lemma 1. $W\left(P_{m, n}, t\right)=\sum_{k=1}^{\min (n, m)}\left(\begin{array}{c}m \\ k\end{array}\right)\left(\begin{array}{l}n \\ k\end{array}\right) t^{k}$.

Proof. Let $\pi=\left(\pi_{1}, \pi_{2}, \ldots\right)$ be a permutation. If $i$ is a descent in $\pi$, we say that $\pi_{i}$ is a descent top and $\pi_{i+1}$ is a descent bottom. Any $\pi \in \mathcal{L}(\mathbf{m} \sqcup \mathbf{n})$ is uniquely determined by its descent tops (which are necessarily elements of $[m+n] \backslash[m]$ ) and descent bottoms (which are elements of $[\mathrm{m}]$ ). It follows that the number of permutations in $\mathcal{L}(\mathbf{m} \sqcup \mathbf{n})$ with exactly $k$ descents is $\left(\begin{array}{c}m \\ k\end{array}\right)\left(\begin{array}{l}n \\ k\end{array}\right)$, implying that $W(\mathbf{m} \sqcup \mathbf{n}, t)=$ $\sum_{k=0}^{\min (n, m)}\left(\begin{array}{c}m \\ k\end{array}\right)\left(\begin{array}{l}n \\ k\end{array}\right) t^{k}$. Since the only element of $\mathcal{L}(\mathbf{m} \sqcup \mathbf{n}) \backslash \mathcal{L}\left(P_{m, n}\right)$ is $(1,2, \ldots, m+n)$, we have $W(\mathbf{m} \sqcup \mathbf{n}, t)=1+W\left(P_{m, n}, t\right)$, and Lemma 1 follows.

We note that all zeros of $W(\mathbf{m} \sqcup \mathbf{n}, t)$ are real and simple (R. Simion [7]).

Proof of Theorem 1. Recall that the Bessel function of order 0 is given by

$$
J_{0}(z)=\frac{2}{\pi} \int_{0}^{1} \frac{\cos (z t)}{\sqrt{1-t^{2}}} d t=\sum_{k=0}^{\infty} \frac{1}{k ! k !}\left(\frac{-z^{2}}{2}\right)^{k} .
$$


It is known that $J_{0}(z)$ has infinitely many zeros, all of which are real and simple. It follows from (2) that $\left|J_{0}(\theta)\right| \leq 1$ for all real $\theta$, with equality only if $\theta=0$. Hence the function

$$
F(z)=\sum_{k=0}^{\infty} \frac{1}{k ! k !} z^{k}
$$

has infinitely many zeros, all of them negative and simple. Also, $|F(\theta)|<1$ for $\theta<0$.

Let $f_{m, n}(z)=W\left(P_{m, n}, z / m n\right)$. Then

$$
f_{m, n}(z)=\sum_{k=1}^{\min (m, n)} \frac{\left(1-\frac{1}{n}\right)\left(1-\frac{2}{n}\right) \cdots\left(1-\frac{k-1}{n}\right)}{k !} \frac{\left(1-\frac{1}{m}\right)\left(1-\frac{2}{m}\right) \cdots\left(1-\frac{k-1}{m}\right)}{k !} z^{k} .
$$

Let $m_{1}, n_{1}, m_{2}, n_{2}, \ldots$ be positive integers such that $\lim _{j \rightarrow \infty} \min \left(m_{j}, n_{j}\right)=\infty$. Then

$$
\lim _{j \rightarrow \infty} f_{m_{j}, n_{j}}(z)+1=F(z),
$$

where the convergence is uniform on any compact subset of $\mathbb{C}$. Let $(-a, 0)$ be an interval containing more than $M$ zeros of $F(z)$. It follows from Hurwitz's theorem [5, Theorem 1.3.8] that the polynomial $f_{m_{j}, n_{j}}(z)+1$ has more than $M$ zeros in $(-a, 0)$ for sufficiently large $j$. By continuity we also have $\left|f_{m_{j}, n_{j}}(z)+1\right|<1$ for $z \in(-a, 0)$ and $j$ large. Thus by subtracting 1 from $f_{m_{j}, n_{j}}(z)+1$, we will lose at least $M$ real zeros.

By applying Sturm's Theorem [5, Section 10.5], one can find specific counterexamples. The polynomial $W\left(P_{11,11}, t\right)$ has two non-real zeros which are approximately

$$
z=-0.10902 \pm 0.01308 i \text {. }
$$

A counterexample with a polynomial of lower degree is

$$
W\left(P_{36,6}, t\right)=216 t+9450 t^{2}+142800 t^{3}+883575 t^{4}+2261952 t^{5}+1947792 t^{6} .
$$

This polynomial has two non-real zeros.

\section{REFERENCES}

[1] P. Brändén, Sign-graded posets, unimodality of $W$-polynomials and the Charney-Davis conjecture, Electr. J. Combin. 11(2), \#9.

[2] F. Brenti, Unimodal, log-concave and Pólya frequency sequences in combinatorics, Mem. Amer. Math. Soc. 413, Amer. Math. Soc., Providence, 1989. MR0963833 (90d:05014)

[3] L. H. Harper, Stirling behavior is asymptotically normal, Ann. Math. Statist. 38 (1967), 410-414. MR0211432 (35:2312)

[4] J. Neggers, Representations of finite partially ordered sets, J. Comb. Inf. Syst. Sci. 3 (1978), 113-133. MR0551484 (58:27668)

[5] Q. I. Rahman and G. Schmeisser, Analytic theory of polynomials, The Clarendon Press, Oxford University Press, Oxford, 2002. MR 1954841 (2004b:30015)

[6] V. Reiner and V. Welker, On the Charney-Davis and Neggers-Stanley conjectures, preprint; available at www.math.umn.edu/ reiner/.

[7] R. Simion, A multiindexed Sturm sequence of polynomials and unimodality of certain combinatorial sequences. J. Comb. Theory, Ser. A 36 (1984), 15-22. MR0728500 (85e:05015)

[8] R. P. Stanley, Ordered structures and partitions, Mem. Amer. Math. Soc. 119, Amer. Math. Soc., Providence, 1972. MF 0332509 (48:10836)

[9] R. P. Stanley, Hilbert functions of graded algebras, Adv. Math. 28 (1978), 57-83. MR0485835 (58:5637) 
[10] R. P. Stanley, Combinatorics and commutative algebra, Birkhäuser Boston Inc., Boston, MA, 2nd edition, 1996. MR 1453579 (98h:05001)

[11] D. Wagner, Enumeration of functions from posets to chains, Eur. J. Comb. 13 (1992), 313324. MR 1179527|(94c:05008)

Department of Mathematics, Chalmers University of Technology and Göteborg UniVERSiTy, S-412 96 GÖTEBorG, SWEDEN

E-mail address: branden@math.chalmers.se 